\title{
MPC-PID control of operator-in-the-loop overhead cranes: A practical approach
}

\author{
Marco Giacomelli - Marco Faroni - Domenico Gorni \\ Alberto Marini - Luca Simoni - Antonio Visioli
}

\begin{abstract}
In this paper, a velocity control system for industrial overhead cranes based on a Model Predictive Control approach is proposed. The problem of the control of the operator-in-the-loop system is addressed, as the operator drives the system pushing a button while the control algorithm drives the cart reducing the oscillations of the load. An inner velocity control loop is used in order to overcome some of the problems of controlling the system by using directly the torque of the motor as a control variable. Simulations show the effectiveness of the approach, in particular in the presence of friction.
\end{abstract}

Index Terms-Overhead crane, MPC, PID, oscillation reduction, operator-in-the-loop.

\section{INTRODUCTION}

Due to their simple design, overhead cranes are the most widespread material handling systems in industry. The presence of persistent payload oscillations during and after the movement, due to the very low damping ratios, makes the manual control difficult, decreasing the accuracy and increasing the overall positioning time. Being underactuated systems, the control of overhead cranes has raised increasing interest in both research and industrial fields in the last thirty years [1], [2]. The main target is the reduction of positioning errors and travelling time in order to increase the productivity and reduce the safety hazard related to the presence of oscillatory heavy loads [3], [4].

In the last decades, several control techniques have been proposed for the control of overhead cranes. They can be roughly classified as open-loop or closed-loop techniques. Open-loop strategies are the most used by far. Their main advantage is that they do not need the presence of sensors measuring the state of the crane (nominally, the payload sway angle). This is an important plus considering that these sensors are usually not present in standard industrial cranes. Moreover, open-loop techniques are based on the model of the process to be controlled, and, given that, a fairly accurate model of an overhead crane is easy to obtain, guaranteeing performance that are enough for most of the industrial applications where overhead cranes are involved. Some of these open-loop techniques are based on optimal control algorithms as in [5], [6]. Furthermore, input-output inversion technique has been proposed for the residual oscillation reduction [7][9]. Input-output inversion consists in the inversion of the transfer function linking the input of the system (e.g., the

M. Giacomelli, M. Faroni, D. Gorni, A. Marini, L. Simoni, and A. Visioli are with the Dipartimento di Ingegneria Meccanica e Industriale, Università degli Studi di Brescia, Italy (e-mail: m.giacomelli009@unibs.it; m.faroni003@unibs.it; domenico.gorni@unibs.it; alberto.marini@unibs.it; luca.simoni@unibs.it; antonio.visioli@unibs.it). force acting on the cart or the velocity of the cart itself) and the output variable of the overhead crane model (usually the velocity/position of the payload). By defining a payload trajectory with given differentiability properties, the output can be computed according to the inverted transfer function. Actually, input shaping techniques [10] are the most widely used in the industrial field for the open-loop control of overhead cranes mainly for two reasons: their ease of implementation on offthe-shelf industrial components and the lack of the need of an accurate model of the system. As a matter of fact, they only rely on the knowledge of the oscillating modes of the system. The aforementioned strategies have also been proposed for the control of overhead cranes with significant double-pendulum dynamics [11]-[13].

Conversely, closed-loop techniques are less diffused in the control of industrial overhead cranes because they require sensors to measure the oscillations of the payload. Furthermore, these strategies require ad-hoc hardware in addition to standard industrial crane layouts. On the other hand, closedloop techniques guarantee an increased degree of robustness with respect to open-loop ones, and the decreasing cost of plug-and-play sensors justifies the increasing interest in their use [14].

In the past years, a number of different closed-loop techniques have been proposed for the control of overhead cranes. For example, adaptive controllers have been devised in [15] [18], while fuzzy control has been proposed in [19]-[21]. Linear and nonlinear techniques have been applied in order to solve the problem of the oscillation of the payload in [22], [23].

During the last twenty years, thanks to the increasing computational power and the reducing cost of industrial PCs, Model Predictive Control (MPC) algorithms have been applied to the control of mechatronic systems which exhibit relatively fast dynamics. The possibility to explicitly include limits and constraints on the output, on the input and on the internal states of the system has proved to be of utter importance in the control of overhead cranes. For example, constraints can be imposed on the maximum load oscillation angle, which is directly correlated with the safety hazard of crane movements. The robustness of MPC techniques on both external disturbances and errors in the model parameters is crucial in an industrial environment. When compared to other closedloop techniques, MPC has been proved to be a competitive solution for the control of overhead cranes [24]. Moreover, the issue of the relatively high computational time, related to the MPC optimization problem, can be the limitation for the real-time implementation of the method [25]. 
MPC has been used for the position control of the payload, with the aim of reducing the oscillation angle [26], [27]. Some of the proposed MPC applications consist in the optimal tracking of a reference trajectory computed offline; in [28] a nonlinear MPC tracks payloads trajectories in a $3 \mathrm{D}$ environment. Changes in the length of the cable are addressed by means of a gain scheduling approach. In [29], [30] payload trajectories are computed by solving an energy consumption reduction optimal problem; these trajectories are then tracked using an MPC algorithm. A method based on MPC for the tracking of 3D trajectories is presented in [31], where computed torque control is used to compensate the modelled nonlinearities of the crane.

The problem of point-to-point motions without an assigned trajectory has also been addressed in [32]-[34]. In [32] a soft constrained MPC approach is proposed and a weight on the sway angle is applied to reduce the residual oscillation. Constraint substitution is used in [33] to convert the online optimization problem into an unconstrained one and thus reducing the computational load of the controller. A similar approach is presented in [34], where the constraints on the sway angle are converted into constraints on the control input. A fast nonlinear MPC based on automatic code generation is proposed in [35].

While the aforementioned closed-loop techniques focus on position control and are thus appropriate for fully automated cranes, little has been done regarding the velocity control of Operator-In-the-Loop (OIL) cranes. Indeed, feedback control with human operated cranes is still an open issue [36]. In fact, while open-loop techniques are well established in the OIL control of overhead cranes, advances in the design and implementation of efficient closed-loop techniques for the velocity control of overhead cranes have a strong relevance from a practical perspective, as these strategies would ensure an increased robustness in the operations with respect to the state-of-the-art open-loop techniques. As a matter of fact, even if the handling of the payload can always be seen as a pointto-point motion, the human operator only has the possibility of pressing one or more buttons. However, there is no means of defining the desired final position of the payload a priori. When the button is pressed, the operator wants the load to reach the steady state velocity and when the button is released the payload shall stop in minimum time, without residual oscillation and without an excessive position overshoot. This means that the resulting control problem can be seen as a velocity-step tracking problem.

Motivated by the increasing computational capacity of standard off-the-shelf control components and the decreasing cost of sensors for feedback controls, in this paper an MPC approach to the problem of OIL overhead cranes control is devised. The MPC approach has already been applied to the velocity control of overhead cranes in [37], and the performance has been compared to input shaping techniques. Therein, at every step, the force acting on the cart is calculated by solving an Optimal Control Problem (OCP) and is given as input to the system. From a practical point of view, a control strategy based ib directly applying a force to the cart presents a few drawbacks. Firstly, standard industrial drives do not always give the possibility to directly control motors in torque; while most of the drives have the option of defining a feedforward torque signal, and velocity and position control loops are always available. Secondly, the need of exploiting a linear model for the optimization problem leads to ignoring the nonlinear friction components (Coulomb friction, viscous friction or temperature dependent friction [38]) acting on the cart, which can be relevant.

In this paper, a modified approach is proposed. Instead of directly controlling the overhead crane by means of the force acting on the cart, as proposed in [37], the velocity of the cart is calculated using the output of the MPC controller (that is, the force acting on the cart) and given as reference to a stiff Proportional-Integral-Derivative (PID) control loop, which is therefore in charge of controlling the velocity of the cart. The force acting on the cart, determined by solving the OCP, is used as a feedforward signal for the velocity loop. This solution leads to improved performance in terms of disturbances rejection (in particular the disturbance introduced by the presence of unmodelled frictions) and robustness with respect to model mismatches (e.g., errors in the estimation of the masses of both cart and payload).

Furthermore, the proposed approach allows a simpler implementation in standard industrial drives, according to the practical considerations exposed above.

The paper is organized as follows. In Section II the model of the overhead crane is presented, its dynamics is described by means of a simple pendulum on a moving cart. Furthermore, in Section III a standard MPC scheme is presented, and an Optimal Control Problem is therefore defined for the control of the previously described model. In Section IV the proposed MPC-PID scheme is described, highlighting the practical advantages that this introduces. In Section $\mathrm{V}$ simulations results show the improvement in performance of the proposed scheme with respect to the standard MPC approach. Finally, Section VI concludes the paper.

\section{MODEL OF THE OVERHEAD CRANE}

An overhead crane can be schematized as a simple pendulum swinging on a sliding moving cart, as shown in Figure 1. The symbols refer to:

$u$ : force applied to the cart;

$m_{C}$ : mass of the cart;

$C_{C}$ : viscous friction coefficient of the cart;

$\theta$ : angle between the vertical and the cable (between cart and payload);

$C_{p}$ : viscous friction coefficient of the cable;

$l \quad$ : length of the cable;

$m_{p}$ : mass of the payload.

With the approximations of a massless inflexible rod and small oscillation angles, a linear model of the crane can be described by the following linear differential equations [37]:

$$
\left\{\begin{array}{l}
\left(m_{p}+m_{C}\right) \ddot{x}+m_{p} l \ddot{\theta}+C_{C} \dot{x}=u \\
m_{p} \ddot{x}+m_{p} l \ddot{\theta}+\frac{C_{p}}{l} \dot{\theta}+m_{p} g \theta=0
\end{array}\right.
$$




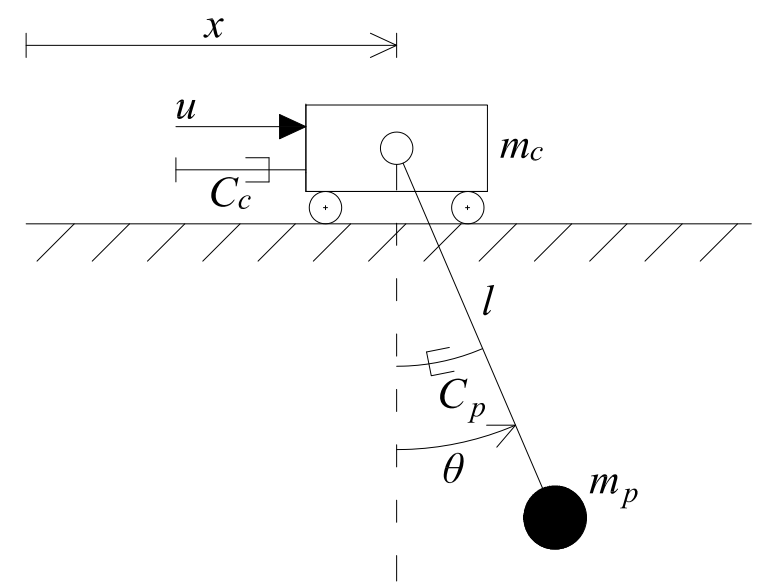

Fig. 1: Scheme of an overhead crane seen as a simple pendulum connected to a sliding cart.

Defining the state vector as $x_{s s}:=(x, \dot{x}, \theta, \dot{\theta})^{T}$ and considering the payload velocity as the output, a state-space description of the system can be obtained as:

$$
\left\{\begin{array}{l}
\dot{x}_{s s}(t)=A x_{s s}(t)+B u(t) \\
y(t)=C x_{s s}(t)
\end{array}\right.
$$

where

$$
\begin{aligned}
A & =\left(\begin{array}{cccc}
0 & 1 & 0 & 0 \\
0 & -\frac{C_{C}}{m_{C}} & \frac{g m_{p}}{m_{C}} & \frac{C_{p}}{m_{C}} \\
0 & 0 & 0 & 1 \\
0 & \frac{C_{C}}{l m_{C}} & -\frac{g\left(m_{p}+m_{C}\right)}{l m_{C}} & -\frac{C_{p}\left(m_{p}+m_{C}\right)}{l^{2} m_{p} m_{C}}
\end{array}\right) \\
B & =\left(\begin{array}{cccc}
0 & \frac{1}{m_{C}} & 0 & -\frac{1}{l m_{C}}
\end{array}\right)^{T} \\
C & =\left(\begin{array}{llll}
0 & 1 & 0 & l
\end{array}\right) .
\end{aligned}
$$

Moreover, an industrial overhead crane is usually subject to hardware and software limitations, nominally the maximum and minimum force that can be applied to the cart ( $u_{\max }$ and $u_{\min }$ respectively), and the maximum and minimum velocity of the cart ( $\dot{x}_{\max }$ and $\dot{x}_{\min }$ respectively).

\section{Model Predictive Control For OIL CRANeS}

As a first approximation, in OIL cranes the operator can drive the payload by means of two buttons: one for the forward movement and one for the backward one. The desired velocity reference $w_{1}$ can be expressed as:

$$
w_{1}:=\left\{\begin{aligned}
\alpha & \text { if forward button is pressed, } \\
-\alpha & \text { if backward button is pressed, } \\
0 & \text { if no button is pressed, }
\end{aligned}\right.
$$

where $\alpha \in \mathbb{R}^{+}$is the desired translational velocity of the payload.

In MPC the optimal input vector (along a given control horizon $N_{C} \in \mathbb{N}$ ) is calculated at each control cycle by solving an OCP over a predictive horizon $N_{P} \in \mathbb{N}$. The control strategy proposed in [37] define the cost function to be minimized in

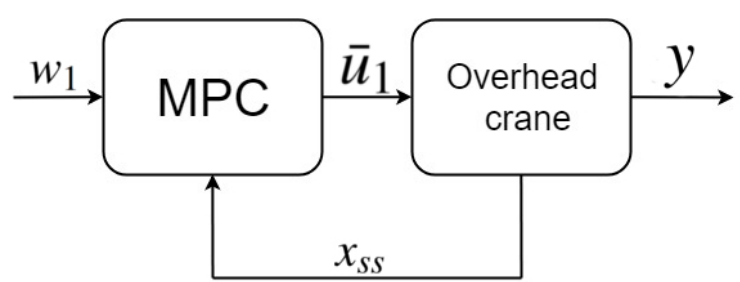

Fig. 2: Standard MPC control scheme.

the OCP as a weighted sum of three terms: i) the payload velocity response; ii) the sway angular velocity; and iii) the control effort. Defining the input vector $\bar{U}:=\left\{\bar{u}_{1}, \ldots, \bar{u}_{N_{c}}\right\}$, the optimal control sequence at time $k$ can be computed as:

$$
\begin{aligned}
\bar{U}=\underset{u}{\operatorname{argmin}} & \sum_{i=1}^{N_{P}}\left(\left.y(k+i)\right|_{k}-w_{1}\right)^{2}+ \\
& +\lambda_{1} \sum_{i=1}^{N_{P}}\left(\left.\dot{\theta}(k+i)\right|_{k}\right)^{2}+\lambda_{u} \sum_{i=0}^{N_{C}-1}(u(k+i))^{2}
\end{aligned}
$$

subject to:

$$
\begin{aligned}
& u_{\min } \leq u(k+i) \leq u_{\max } \quad \forall i \in 1, \ldots, N_{C}-1 \\
& \dot{x}_{\min } \leq\left.\dot{x}(k+i)\right|_{k} \leq \dot{x}_{\max } \quad \forall i \in 1, \ldots, N_{P}
\end{aligned}
$$

where $\lambda_{1} \in \mathbb{R}^{+}$is the weight on the angular velocity of the swaying load, $\lambda_{u} \in \mathbb{R}^{+}$is the weight on the control action, and $\left.y(k+i)\right|_{k},\left.\dot{\theta}(k+i)\right|_{k}$, and $\left.\dot{x}(k+i)\right|_{k}$ are the predictions of $y, \dot{\theta}$, and $\dot{x}$ at time $k$, which are derived from the system matrices $A, B$, and $C$, as typical of linear MPC [39]. The first value of the control vector is then given as input to the system (i.e., $u(k)=\bar{u}(1)$ ) and at the following control time instant the procedure is repeated. This control strategy is sketched in Figure 2.

\section{MPC-PID APPROACH}

The alternative control scheme proposed in this paper is shown in Figure 3.

The overhead crane model described by (2) is linear and, obviously, it is an approximation of the real system. This linear model has been used mainly for two reasons: its good level of approximation for small oscillation angles, and the possibility to apply linear MPC strategies as the one exposed in Section III. However, the presence of unmodelled dynamics (e.g., the Coulomb friction of the cart) and modelling errors (e.g. errors in the estimation of the mass of both cart and payload) decreases the effectiveness of the standard linear MPC approach. An alternatice control scheme is therefore proposed. The idea behind this scheme is that a stiff PID control of the cart velocity can tackle the above-mentioned issues. Therefore, an outer MPC controller is in charge of calculating the optimal cart velocity reference signal $\overline{\dot{x}}$, which has to be followed by the PID inner-loop. Such velocity reference can be computed as the cart velocity predicted at time $k+1$ by applying the optimal control action $u(k)=\bar{u}_{1}$ computed the OCP defined in (7).

In order to increase the performance of the control loop, the optimal force value $\bar{u}_{1}$ is provided as a feedforward signal to the system. Therefore, the PID controller is tuned for 


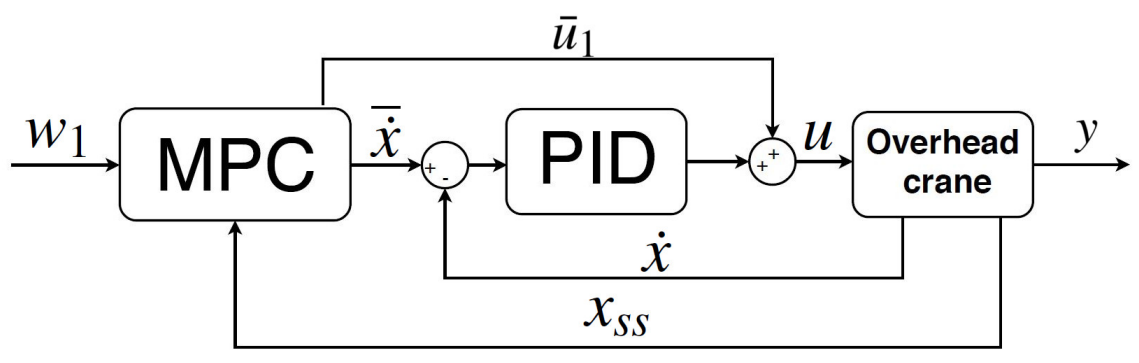

Fig. 3: Proposed MPC-PID control scheme.

disturbance rejection, as its task is to compensate for model uncertainties, external disturbances, and nonlinearities.

It is worth stressing that the use of a PID controller has a strong practical advantage with respect to the standard MPC approach shown in Figure 2. As a matter of fact, off-the-shelf industrial drives do not usually give the possibility to control the motors by directly providing a value to the motor torque, so that a scheme like the one shown in Figure 2 could not be exploitable in practice. Furthermore, standard industrial drives give the user the possibility to tune position and velocity loops, and most of them have an optional torque feedforward signal, making the present approach readily implementable.

\section{Simulations Results}

The effectiveness of the MPC-PID control scheme is evaluated in a comparison test. Both the methodologies (the standard MPC [37] and the proposed MPC-PID approach) have been applied on a nonlinear model of an overhead crane in Simulink, built with the Simscape Multibody environment.

The parameters used for the linear model implemented on the MPC controller are listed in Table I.

TABLE I: Parameters of the model used in simulations.

\begin{tabular}{cc}
\hline Parameter & Value \\
\hline$m_{C}$ & $38[\mathrm{~kg}]$ \\
$C_{C}$ & $20[\mathrm{Ns} / \mathrm{m}]$ \\
$C_{p}$ & $0.1[\mathrm{Nms} / \mathrm{rad}]$ \\
$l$ & $1.5[\mathrm{~m}]$ \\
$m_{p}$ & $10[\mathrm{~kg}]$ \\
\hline
\end{tabular}

The cart velocity and force limits are set equal to

$$
\begin{aligned}
& \dot{x}_{\max }=-\dot{x}_{\min }=1.2[\mathrm{~m} / \mathrm{s}] \\
& u_{\max }=-u_{\min }=1000[\mathrm{~N}]
\end{aligned}
$$

while the weighting parameters of the OCP (7) are set equal to $\lambda_{1}=5.0 \cdot 10^{-4}, \lambda_{u}=3.0 \cdot 10^{-6}$. The velocity setpoint $w_{1}$ is set equal to $\alpha=1.0[\mathrm{~m} / \mathrm{s}]$ when the command button is pressed. The MPC controller sampling period has been set equal to $T_{S_{M P C}}=0.024$ [s] with a prediction horizon of $N_{P}=30$ and a control horizon of $N_{C}=5$. The chosen sampling period is actually reasonable in case of an MPC algorithm directly employed in industrial controllers. The discrete PID controller has a sampling period of $T_{S_{P I D}}=0.001[s]$, a proportional gain $K_{p}=1800$, an integral time constant $T_{i}=0.2778[s]$, a derivative time constant $T_{d}=0.0056[s]$ and a filtering coefficient $N=20$.

In order to include uncertainties in the model parameters, the nonlinear model implemented in Simulink has the same parameters of Table I except for the mass of the cart and the friction coefficient acting on the cart, which are set equal to $m_{C_{R}}=49.4[\mathrm{~kg}]$ and $C_{C_{R}}=10[\mathrm{Ns} / \mathrm{m}]$. Moreover, a Coulomb friction has been added on the cart, with a value of $C_{0}=150$ $[N]$.

During the simulation, at time $t=1[s]$ the button is pressed, and, then, at time $t=6[s]$ the button is released, resulting in the velocity reference shown in Figure 4.

In Figure 6 the load velocities resulting from the application of the standard MPC scheme and the proposed MPC-PID scheme are compared. It can be seen that the performance, in terms of oscillations around the set-point value, of the MPCPID control scheme are improved mainly due to the increased robustness with respect to the introduced errors in the cart mass and friction. Moreover, also the disturbance on the force acting on the cart is rejected with a faster dynamics.

In addition, the proposed scheme guarantees reduced residual oscillation with respect to the standard MPC approach (see Figure 7). It has to be highlighted that, due to the relevant Coulomb friction inserted in the model, residual oscillation are still present in both cases.

The improved performance of the proposed method can be observed also by analyzing Figure 8 where the velocity of the cart is shown for both the schemes. The residual oscillation of the cart is reduced thanks to the cart velocity PID controller.

In Figure 9 the force acting on the cart deriving by the control algorithms is shown (the force calculated by the MPC in one case, the sum of the feedforward force calculated by the MPC and the output of the PID in the other). The values of the two forces are comparable. The main difference can be identified in the fact that the PID has a sampling period of 0.001 [s], while, due to the computational complexity of the OCP, the MPC updates the force every $0.024[s]$. Moreover, at time $t=9[\mathrm{~s}]$ a constant positive disturbance of $500[N]$ is added on the force acting on the cart (see Figure 5). 


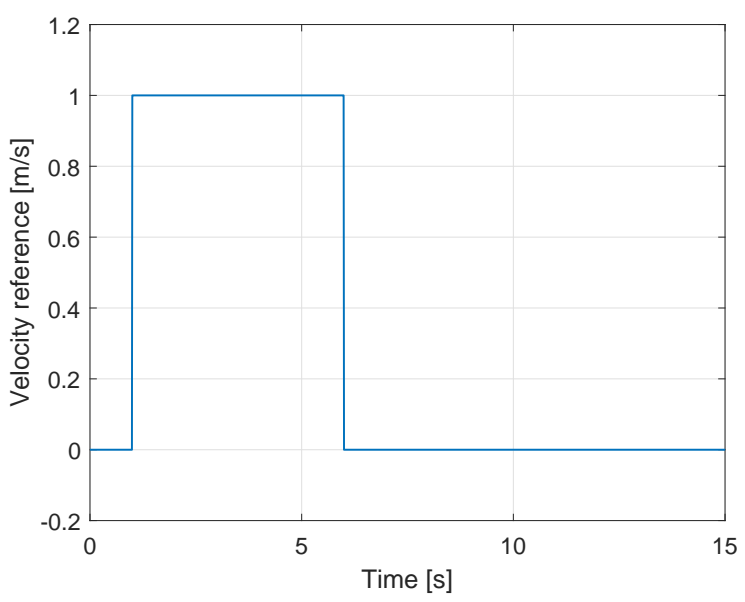

Fig. 4: Payload velocity reference.

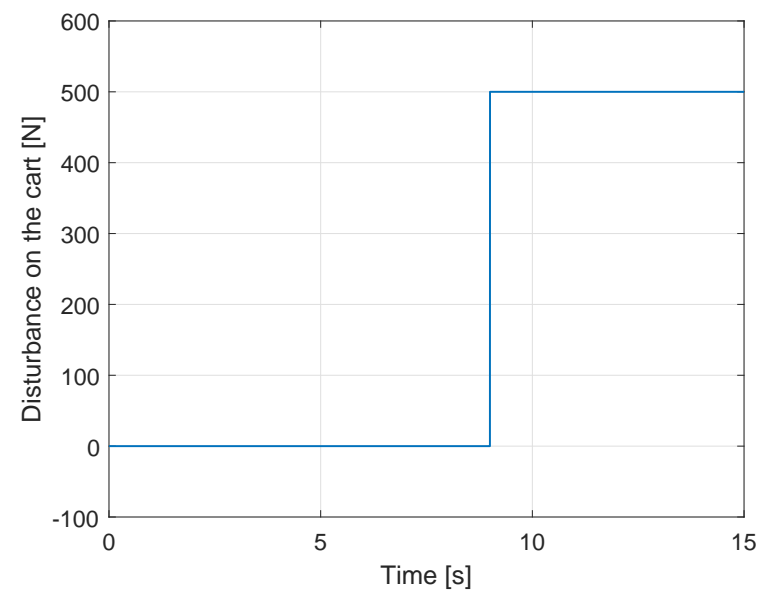

Fig. 5: Disturbance acting on the force acting on the cart at time $t=9[s]$.

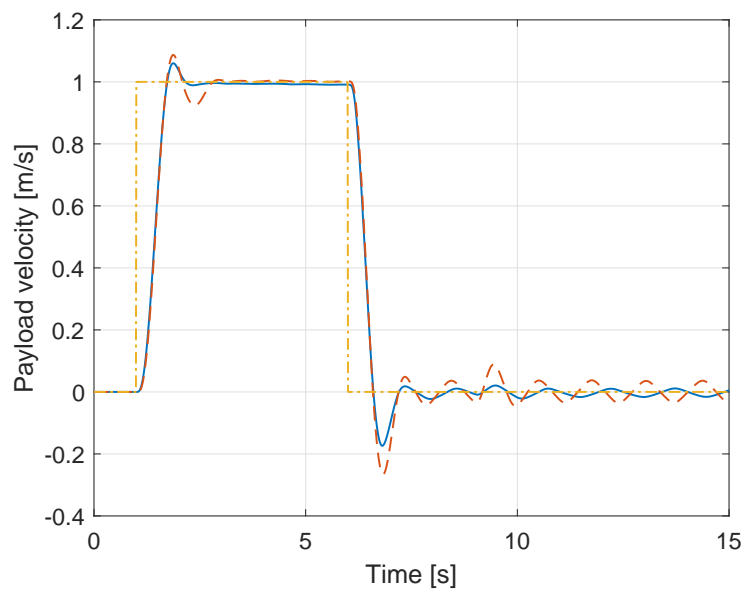

Fig. 6: Load velocity response of the MPC standard scheme (red dashed line) and the MPC-PID scheme (blue solid line). The velocity reference $w_{1}$ is the yellow dash-dot line.

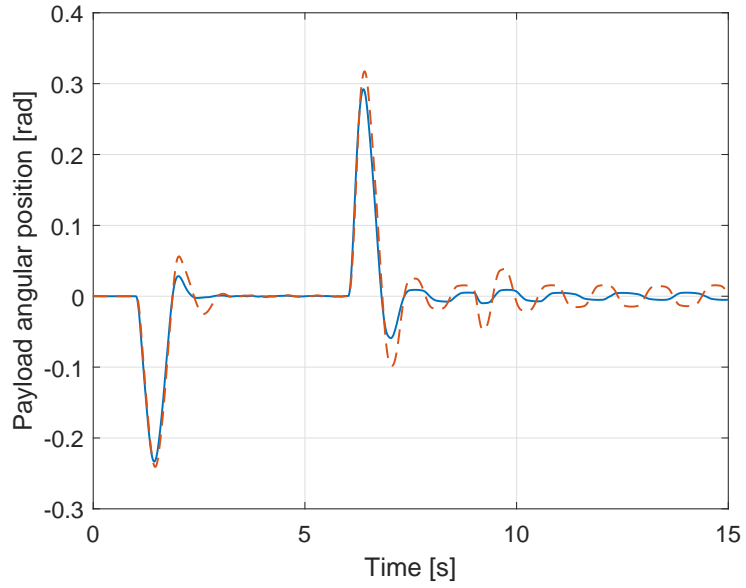

Fig. 7: Angular position $\theta$ in case of standard MPC control scheme (red dashed line) and in case of the proposed MPCPID approach (blue solid line).

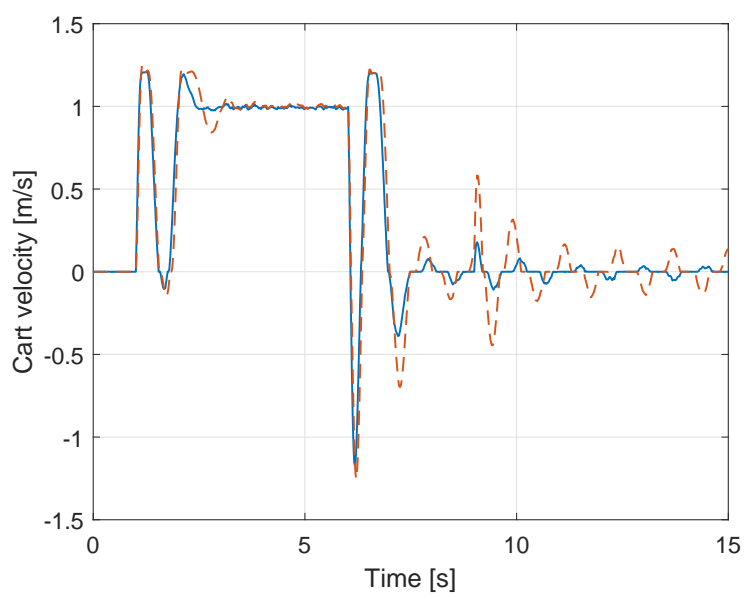

Fig. 8: Cart velocity $\dot{x}$ for both standard MPC (red dashed line) and MPC-PID control schemes (blue solid line).

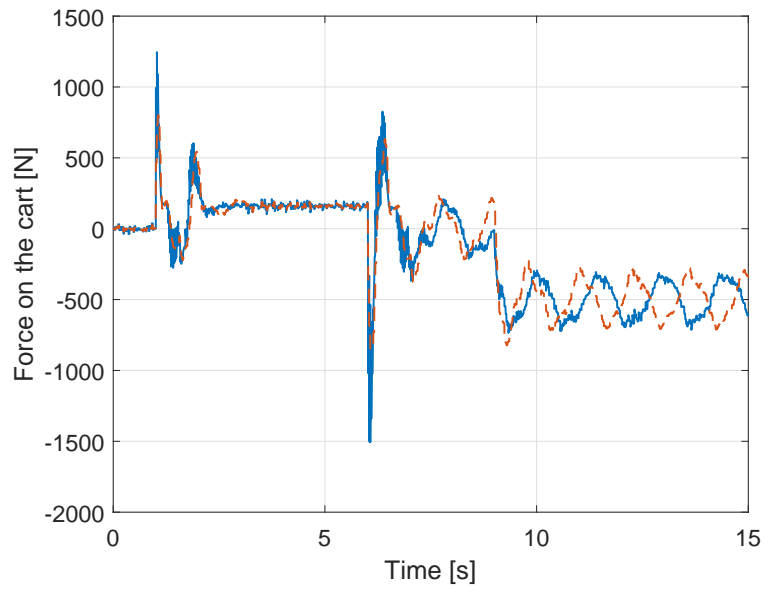

Fig. 9: Control force acting on the cart. Standard MPC control (dashed red line), MPC-PID scheme (blue solid line) Notice that, in this case, the force is given by the sum of the feedforward force and the output of the PID. 


\section{CONCLUSiOnS}

In this paper, a practical MPC-PID approach has been proposed for the velocity control of operator-in-the-loop overhead cranes. The proposed method has been proved to effectively improve the performance with respect to the standard MPC scheme proposed in [37], in terms of reduced oscillation during the movement and reduced residual oscillation in case of mismatches in the model parameters (mass of the cart, friction, nonlinearities). Future works will focus on the implementation of the proposed technique on an industrial overhead crane, proving the feasibility of the proposed scheme with industrial off-the-shelf components. Moreover, the problem of the residual oscillation due to the presence of Coulomb friction has to be addressed for the proposed scheme to be an efficient alternative to the state of the art techniques.

\section{ACKNOWLEDGEMENTS}

This work has been developed within the European Union H2020 program ECSEL-2016-1 under grant agreement $n$. 737453 (I-MECH).

\section{REFERENCES}

[1] E. M. Abdel-Rahman, A. H. Nayfeh, and Z. N. Masoud, "Dynamics and control of cranes: A review," Modal Analysis, vol. 9, no. 7, pp. 863-908, 2003.

[2] P. Hyla, "The crane control systems: A survey," in Prooceedings of the IEEE International Conference on Methods and Models in Automation and Robotics, 2012, pp. 505-509.

[3] X. Zhang, Y. Fang, and N. Sun, "Minimum-time trajectory planning for underactuated overhead crane systems with state and control constraints," IEEE Transactions on Industrial Electronics, vol. 61, no. 12, pp. 6915-6925, 2014.

[4] J. Auernig and H. Troger, "Time optimal control of overhead cranes with hoisting of the load," Automatica, vol. 23, no. 4, pp. 437-447, 1987.

[5] J. A. Field, "The optimization of the performance of an ore bridge," Transactions of the Engineering Institute of Canada, vol. 5, no. 3, pp. 163-169, 1961.

[6] A. Al-Garni, K. Moustafa, and S. J. Nizami, "Optimal control of overhead cranes," Control Engineering Practice, vol. 3, no. 9, pp. 12771284, 1995.

[7] A. Piazzi and A. Visioli, "Minimum-time system-inversion-based motion planning for residual vibration reduction," IEEE/ASME Transactions on Mechatronics, vol. 5, no. 1, pp. 12-22, 2000.

[8] F. Padula, A. Visioli, D. Facchinetti, and A. Saleri, "A dynamic inversion approach for oscillation-free control of overhead cranes," in Proceedings of the IEEE International Conference on Emerging Technologies and Factory Automation, 2015, pp. 1-6.

[9] A. Piazzi and A. Visioli, "Optimal dynamic-inversion-based control of an overhead crane," IEE Proceedings-Control Theory and Applications, vol. 149, no. 5, pp. 405-411, 2002.

[10] W. Singhose, "Command shaping for flexible systems: A review of the first 50 years," International Journal of Precision Engineering and Manufacturing, vol. 10, no. 4, pp. 153-168, 2009.

[11] W. E. Singhose, D. Kim, and M. Kenison, "Input shaping control of double-pendulum bridge crane oscillations," Journal of Dynamic Systems, Measurement, and Control, vol. 130, no. 3, 2008.

[12] M. Giacomelli, F. Padula, L. Simoni, and A. Visioli, "Simplified inputoutput inversion control of a double pendulum overhead crane for residual oscillations reduction," Submitted to Mechatronics.

[13] N. Sun, Y. Wu, H. Chen, and Y. Fang, "An energy-optimal solution for transportation control of cranes with double pendulum dynamics: Design and experiments," Mechanical Systems and Signal Processing, vol. 102, pp. 87-101, 2018.

[14] H. M. Omar and A. Nayfeh, "Anti-swing control of gantry and tower cranes using fuzzy and time-delayed feedback with friction compensation," Shock and Vibration, vol. 12, no. 2, pp. 73-89, 2005.

[15] J. Ackermann, "Parameter space design of robust control systems," IEEE Transactions on Automatic Control, vol. 25, no. 6, pp. 1058-1072, 1980.
[16] A. Marttinen, "Pole-placement control of a pilot gantry," in Proceedings of the American Control Conference, 1989, pp. 2824-2826.

[17] R. Salminen, A. Marttinen, and J. Virkkunen, "Adaptive pole placement control of a pilot crane," IFAC Proceedings Volumes, vol. 23, no. 8, pp. 313-318, 1990

[18] J. M. Virkkunen and A. T. Marttinen, "Computer control of a loading bridge," in Proceedings of the International Conference on Control, 1988, pp. 484-488.

[19] H. Oshima, S. Yasunobu, and S. Sekino, "Automatic train operation system based on predictive fuzzy control," in Artificial Intelligence for Industrial Applications, Proceedings of the International Workshop on, 1988, pp. 485-489.

[20] S. Yasunobu and T. Hasegawa, "Evaluation of an automatic container crane operation system based on predictive fuzzy control," Controltheory and advanced technology, vol. 2, no. 3, pp. 419-432, 1986.

[21] _ "Predictive fuzzy control and its application for automatic container crane operation system," in Proceedings of the 2nd. IFSA Congress, 1987

[22] H. Lee, S. Cho, and J. Cho, "A new anti-swing control of overhead cranes," IFAC Proceedings Volumes, vol. 30, no. 13, pp. 115-120, 1997.

[23] Y. Fang, W. Dixon, D. Dawson, and E. Zergeroglu, "Nonlinear coupling control laws for an underactuated overhead crane system," IEEE/ASME Transactions on Mechatronics, vol. 8, no. 3, pp. 418-423, 2003.

[24] J. Smoczek and J. Szpytko, "Comparision of model predictive, input shaping and feedback control for a lab-scaled overhead crane," in Proceedings of the International Conference on Methods and Models in Automation and Robotics, 2016, pp. 288-293.

[25] K. Graichen, M. Egretzberger, and A. Kugi, "Suboptimal model predictive control of a laboratory crane," IFAC Proceedings Volumes, vol. 43, no. 14, pp. 397-402, 2010.

[26] L. Van den Broeck, M. Diehl, and J. Swevers, "Experimental validation of time optimal MPC on a flexible motion system," in Proceedings of the American Control Conference, pp. 4749-4754.

[27] J. Smoczek and J. Szpytko, "Soft-constrained predictive control for an overhead crane," Journal of KONES Powertrain and Transport, vol. 24, no. 3, 2017.

[28] D. Schindele and H. Aschemann, "Fast nonlinear MPC for an overhead travelling crane," IFAC Proceedings Volumes, vol. 44, no. 1, pp. 7963 7968, 2011.

[29] Z. Wu, X. Xia, and B. Zhu, "Model predictive control for improving operational efficiency of overhead cranes," Nonlinear Dynamics, vol. 79 no. 4, pp. 2639-2657, 2015.

[30] Z. Wu and X. Xia, "Energy efficiency of overhead cranes," IFAC Proceedings Volumes, vol. 47, no. 3, pp. 19-24, 2014.

[31] A. Khatamianfar and A. V. Savkin, "A new tracking control approach for $3 \mathrm{~d}$ overhead crane systems using model predictive control," in Proceedings of the European Control Conference, pp. 796-801.

[32] D. Jolevski and O. Bego, "Model predictive control of gantry/bridge crane with anti-sway algorithm," Journal of mechanical science and technology, vol. 29, no. 2, pp. 827-834, 2015.

[33] B. Käpernick and K. Graichen, "Model predictive control of an overhead crane using constraint substitution," in Proceedings of the American Control Conference, 2013, pp. 3973-3978.

[34] H. Chen, Y. Fang, and N. Sun, "A swing constraint guaranteed MPC algorithm for underactuated overhead cranes," IEEE/ASME Transactions on Mechatronics, vol. 21, no. 5, pp. 2543-2555, 2016.

[35] M. Vukov, W. Van Loock, B. Houska, H. J. Ferreau, J. Swevers, and M. Diehl, "Experimental validation of nonlinear MPC on an overhead crane using automatic code generation," in Proceedings of the American Control Conference, 2012, pp. 6264-6269.

[36] L. Ramli, Z. Mohamed, A. M. Abdullahi, H. Jaafar, and I. M. Lazim, "Control strategies for crane systems: A comprehensive review," Mechanical Systems and Signal Processing, vol. 95, pp. 1-23, 2017.

[37] M. Giacomelli, M. Faroni, D. Gorni, A. Marini, L. Simoni, and A. Visioli, "Model predictive control for operator-in-the-loop overhead cranes," in Proceedings of the IEEE International Conference on Emerging Technologies and Factory Automation.

[38] L. Simoni, M. Beschi, G. Legnani, and A. Visioli, "Modelling the temperature in joint friction of industrial manipulators," Robotica, p. 122, 2017

[39] L. Wang, Model predictive control system design and implementation using MATLAB®. Springer Science \& Business Media, 2009. 